Check for updates

Cite this: RSC Adv., 2019, 9, 15350

\title{
Dansyl acetyl trehalose: a novel tool to investigate the cellular fate of trehalose $\uparrow$
}

\author{
Alessandra Locatelli, $\dot{t}^{a}$ Luisa Iommarini, $\neq^{a}$ Alessandra Graziadio, ${ }^{a}$ Alberto Leoni, ${ }^{a}$ \\ Anna Maria Porcelli, ${ }^{\text {ab }}$ Stefano lotti, (D) ac Emil Malucelli, ${ }^{a}$ Francesco Francia, ${ }^{a}$ \\ Giovanni Venturolia and Giovanna Farruggia (D) $\mathbf{t}^{\text {*ac }}$
}

Received 9th March 2019

Accepted 19th April 2019

\begin{abstract}
A fluorescent derivative of trehalose with two dansyl groups (DAT) has been synthesized. It is characterised by a large Stokes shift, good permeability in human living cells and a well detectable fluorescent signal within the cells. Notably, in intestinal cells DAT is sequestered in vesicles induced by trehalose pretreatment and colocalizes with lipid droplets.
\end{abstract}

rsc.li/rsc-advances

Trehalose is a disaccharide formed by two molecules of Dglucose linked by an $\alpha, \alpha-1,1$ bond. It occurs in prokaryotes and eukaryotes, but vertebrates lack the synthetic pathway for this sugar. Trehalose has several important functions: for instance, in insects, it represents an important energy source and the target of different signalling pathways mediated by nutrients; in plants, it is widely present, and its synthetic pathway is a sensor of the glucose availability. This sugar is also widely known for its role in organisms, like yeast, rotifers, nematodes and tardigrades, which have to cope with harsh environmental conditions, such as extreme dryness or freezing. ${ }^{1}$ The interest for the use of this sugar as a bioprotectant raised in the recent past, and, in particular, trehalose-based protocols have been devised aimed to the cryo- or lyo-preservation of mammalian cells. ${ }^{2-6}$

To exert its bioprotective effects, trehalose has to be localized both in extra- and intra-cellular compartments. In this regard, its delivery within the cells represents a challenge, because mammalian cells lack a specific transporter for this sugar. It has been proposed that the glucose carrier GLUT8 could efficiently transport this sugar, but this membrane protein has been reported to be scarcely present in mammalian cells. ${ }^{7}$ Several strategies have been proposed to overcome this hurdle, including electropermeabilization, ${ }^{8}$ overexpression in mammalian cells of the trehalose transporter (TRET1) from the anhydrobiotic larvae of the African chironomid Polypedilum vanderplanki, ${ }^{9}$ and freezing-induced osmotic stress in the presence of trehalose. ${ }^{3}$ Recently the synthesis of an engineered trehalose obtained by conjugating its hydroxyls with six acetyl

\footnotetext{
aDipartimento di Farmacia e Biotecnologie (FABIT), Università degli Studi di Bologna, 40127, Bologna, Italy. E-mail: Giovanna.Farruggia@unibo.it

${ }^{b}$ Centro Interdipartimentale di Ricerca Industriale (CIRI) Scienze della Vita $e$ Tecnologie per la Salute, Università di Bologna, 40100 Bologna, Italy

${ }^{c}$ National Institute of Biostructures and Biosystems, Roma 00136, Italy

$\dagger$ Electronic supplementary information (ESI) available. See DOI: $10.1039 / \mathrm{c} 9 \mathrm{ra} 01800 \mathrm{j}$

$\$$ These authors contribute equally to this work.
}

groups has been reported. ${ }^{10}$ This molecule is significantly more permeable than trehalose in rat hepatocytes; furthermore, once in the cells, intracellular esterases rapidly reconvert the acetylated form to trehalose, increasing its intracellular concentration to values ten-fold larger than the extracellular concentration of the acetyl-trehalose.

In this context, the availability of fluorescent derivatives could represent an important tool to follow the intracellular fate of trehalose. However, only few trehalose fluorescent derivatives have been published so far, mainly employed in microbiological studies. ${ }^{11,12}$

The aromatic 1,1-dimethylaminonaphthalene-5-sulfonyl chloride, or dansyl chloride (DNS-Cl), represents a fluorophore with very favourable properties because of its high fluorescence quantum yield and large Stokes shift. It is widely used in peptide chemistry, and in particular as a chromatographic derivatization reagent to identify several kinds of analytes. ${ }^{13-18}$

In this paper, we describe the synthesis of dansyl acetyl trehalose (DAT), examine its spectroscopic properties, and demonstrate for the first time that it can be successfully applied to detect trehalose uptake and compartmentalization in human intestinal cell lines in specific vesicles.

The first step of our synthetic strategy (see Fig. 1) is the protection of the two primary alcoholic functions with a trityl group using triphenylmethyl chloride or trityl chloride ( $\mathrm{TrCl})$ as described in the literature, ${ }^{19}$ to give compound 1 . The trityl group has been frequently used for the temporary protection of primary hydroxyl groups for its easy removal by treatment with weak acid. ${ }^{20}$ For the protection of secondary alcohols, it has been decided to use acetyl groups instead of benzyl groups, obtaining compound 2; the deprotection of the acetyl groups results, in fact, in addition to be simpler, faster and more efficient. Trityl deprotection of compound 2 was accomplished with iron chloride hexahydrate $\left(\mathrm{FeCl}_{3} \cdot 6 \mathrm{H}_{2} \mathrm{O}\right)$ in methylene chloride to yield trehalose hexaacetate (compound 3 ). It has 


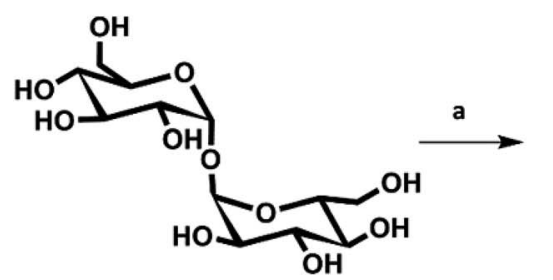

Trehalose

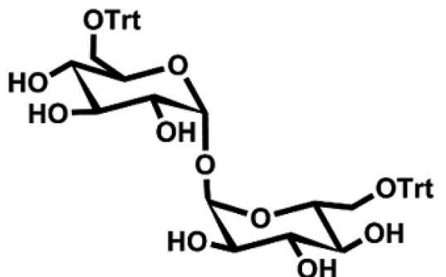

1

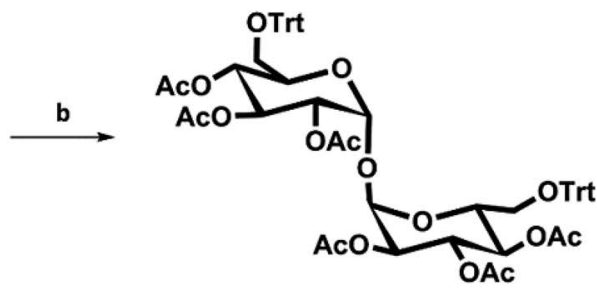

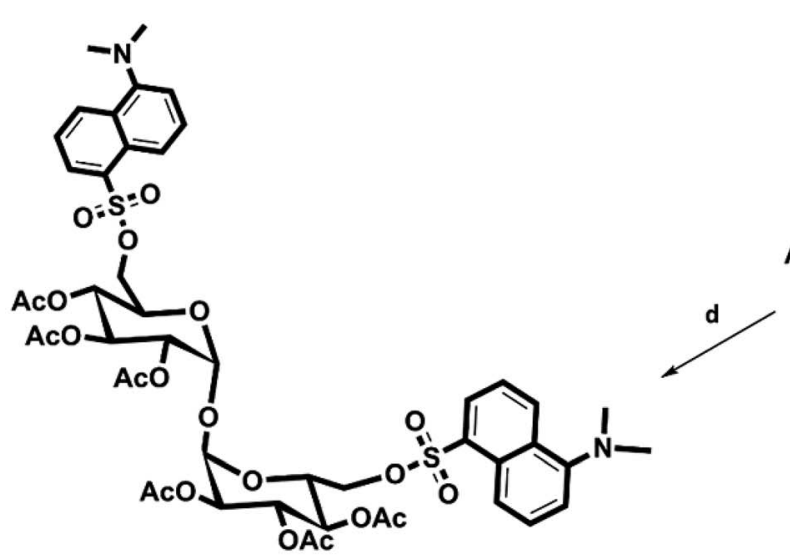

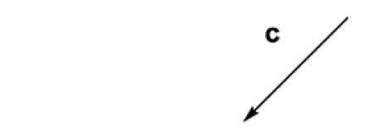

4
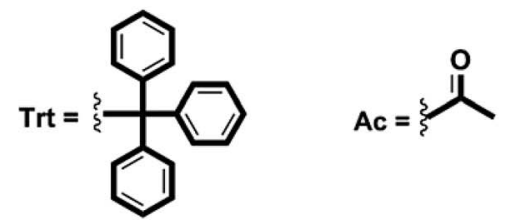

Fig. 1 Scheme of DAT synthesis. Reagents: (a) $\mathrm{TrCl}$, py, $75^{\circ} \mathrm{C}$; (b) $\mathrm{Ac}_{2} \mathrm{O}, \mathrm{TEA}, \mathrm{DMAP}, \mathrm{RT}$; (c) $\mathrm{FeCl}_{3} \cdot 6 \mathrm{H}_{2} \mathrm{O}, \mathrm{CH}_{2} \mathrm{Cl}_{2}, \mathrm{RT}$; (d) DNS-Cl, TEA, DMAP, dry $\mathrm{CH}_{2} \mathrm{Cl}_{2}, \mathrm{RT}$.

been reported that iron chloride hexahydrate is an effective reagent for detritylation..$^{20}$ With this reagent, in fact, there were no cases of acyl migrations, breakdown of glycosidic bonds, or anomerizations. Finally, the dansylation reaction was carried out, under nitrogen atmosphere, to obtain compound 4 (DAT).

We have assessed the spectrofluorimetric properties of DAT, by examining the effect of solvent polarity on excitation and emission spectra. In fact, it is well known that solvent polarity (static dielectric constant) can heavily affect fluorescence, ${ }^{21}$ and DNS-Cl in particular responds to different environmental conditions. ${ }^{17}$ The results are reported in Fig. 2, which compares DAT (panels A and B) with DNS-Cl (panels C and D) spectra. Table 1 summarizes the peak wavelengths $\left(\lambda_{\mathrm{Em}}\right.$ and $\left.\lambda_{\mathrm{Ex}}\right)$ of the emission and excitation spectra, respectively.

DNS-Cl fluorescence is heavily modulated by the solvent dielectric constant $\varepsilon$ : upon increasing polarity, the emission spectra exhibit a red shift, with the peak moving from $423 \mathrm{~nm}$ in acetone to $495 \mathrm{~nm}$ in DPBS. Such an effect is not observed in the excitation spectra, the peaks of which range from 320 to $326 \mathrm{~nm}$, with the exception of acetone, for which the excitation maximum is at $333 \mathrm{~nm}$.

Interestingly, chloroform completely turns off DNS-Cl fluorescence. When dansyl is bound to acetyl trehalose, however, it loses this sensitivity to solvent polarity, as shown by the spectra reported in Fig. 2A and B. The emission and excitation peaks lay in a narrow range of wavelengths, from 500 to $526 \mathrm{~nm}$, and from 350 to $356 \mathrm{~nm}$, respectively. Both excitation and emission spectra of DAT are significantly red shifted in comparison to those of DNS-Cl.

In addition, the influence of the tested solvents on the fluorescence intensity is quite different in DNS-Cl and DAT: as shown in Fig. 2, panel D, DNS-Cl fluorescence emission is higher in ethanol and methanol than in aqueous solution or in acetone, being completely turned off in chloroform. On the contrary, DAT shows the highest emission in water and in chloroform and the ionic strength of the physiological saline solution DPBS faints just a little bit the fluorescence (Fig. 2, panel B). Acetone, ethanol and methanol progressively decrease the fluorescence intensity. Because of these spectral characteristics, DAT can be considered a good fluorescent dye suitable for the study of trehalose uptake and compartmentalization in cells. In aqueous solvents, as DPBS or culture medium, DAT shows $\lambda_{\mathrm{Ex}}$ centred around $350 \mathrm{~nm}$ and $\lambda_{\mathrm{Em}}$ around $500 \mathrm{~nm}$, features that allow designing applications in fluorescence microscopy or flow cytometry. Indeed, the broad emission spectra allow detecting this dye both in blue and green emission 

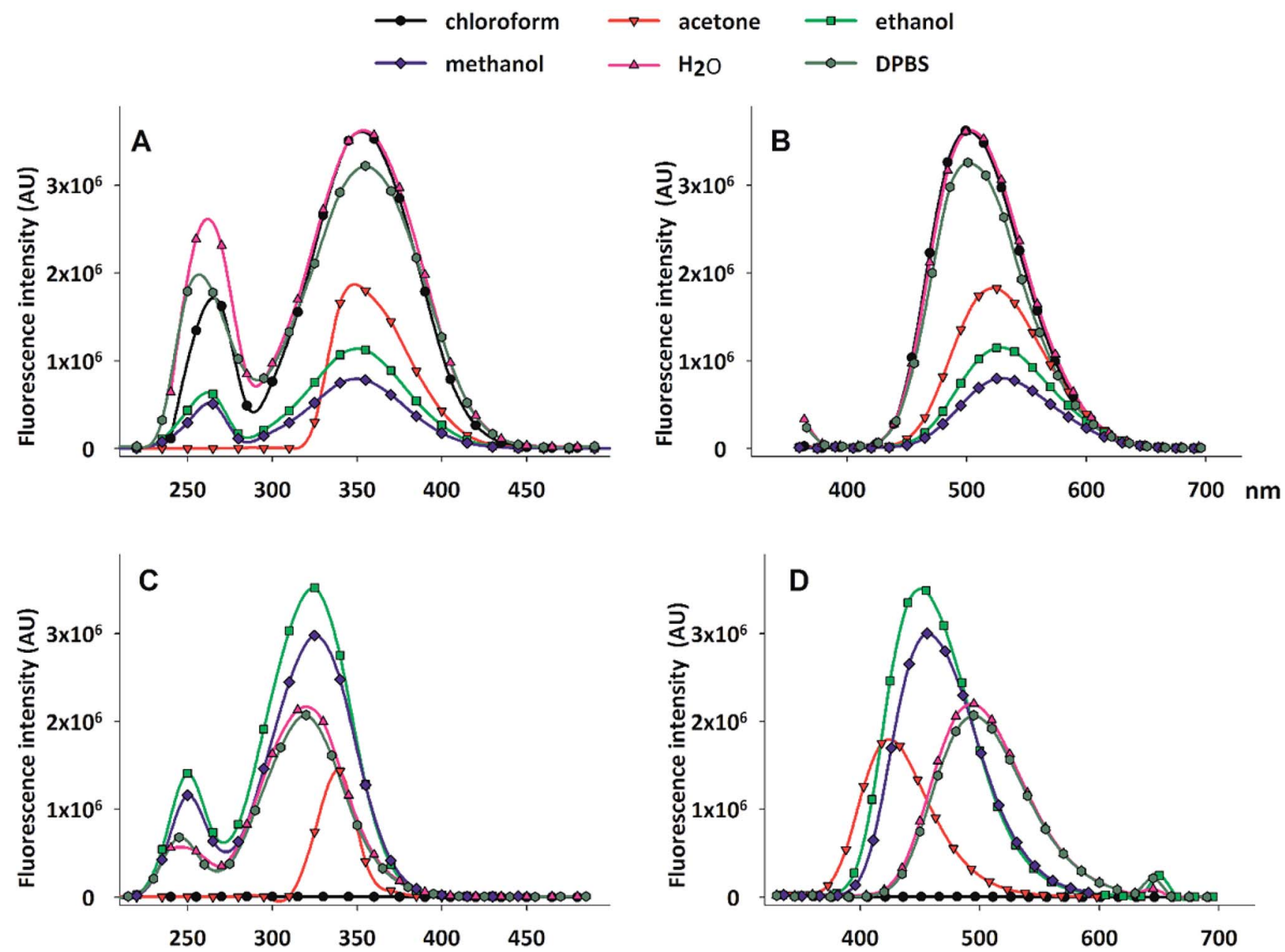

Fig. 2 Fluorescence spectra of DAT (A, B) and DNS-Cl (C, D) in different solvents. (A) and (C): excitation spectra; (B) and (D): emission spectra. $10 \mathrm{mM}$ stock solutions of DAT and DNS-Cl in DMSO were dissolved in the reported solvents to a $10 \mu \mathrm{M}$ final concentration. The spectra were acquired as detailed under Materials and methods in the ESI. $\dagger$

Table $1 \lambda_{E m}$ and $\lambda_{E x}$ of DAT and DNS-Cl in solvents characterized by different dielectric constant $(\varepsilon)$ values

\begin{tabular}{|c|c|c|c|c|c|}
\hline \multirow[b]{2}{*}{ Solvent } & \multirow[b]{2}{*}{$\varepsilon$} & \multicolumn{2}{|l|}{ DAT } & \multicolumn{2}{|l|}{ DNS-Cl } \\
\hline & & $\lambda_{\mathrm{Em}}(\mathrm{nm})$ & $\lambda_{\mathrm{Ex}}(\mathrm{nm})$ & $\lambda_{\mathrm{Em}}(\mathrm{nm})$ & $\lambda_{\mathrm{Ex}}(\mathrm{nm})$ \\
\hline Chloroform & 4.8 & 500 & 353 & - & - \\
\hline Acetone & 21 & 521 & 350 & 423 & 333 \\
\hline Ethanol & 24 & 526 & 350 & 451 & 321 \\
\hline Methanol & 33 & 520 & 350 & 455 & 326 \\
\hline $\mathrm{H}_{2} \mathrm{O}$ & 80 & 500 & 356 & 488 & 320 \\
\hline DPBS & 80 & 501 & 354 & 495 & 321 \\
\hline
\end{tabular}

fields. The weak influence of polarity permit the detection of DAT fluorescence in all the compartments of the cell, independently from their hydrophobicity.

Furthermore, DAT exhibits a large Stokes shift between excitation and emission wavelengths (see Fig. 2 and Table 1), which makes it an excellent probe in fluorescence microscopy, especially when spectral separation is required to reduce fluorescence overlap.
When we examined the cellular uptake of DAT, it was mandatory to reduce the concentration of the fluorescent sugar added to the cells, because in culture medium, at concentrations higher than $5 \mu \mathrm{M}$, the dye aggregates. Furthermore, to ameliorate the dispersion of the dye in culture medium, the solution was set in a sonication bath for $3 \mathrm{~min}$ immediately before use. This treatment does not influence the fluorescence performance of the dye (data not shown). Incubation of three different colon cancer cell lines (LoVo, RKO and HCT116) with 1 $\mu \mathrm{M}$ DAT for 30 minutes showed a dotted pattern, that was mainly localized in body/vesicle-like structures, similar to those described by Higuchi (Fig. 3A). ${ }^{22}$ On the contrary, cells incubated with $1 \mu \mathrm{M}$ DNS-Cl showed a faint extracellular diffused fluorescence (Fig. 3B), indicating that only DAT is specifically compartmentalized into the cells. Interestingly, HCT116 displayed very few DAT-positive intracellular vesicles compared to LoVo and RKO cells.

It has been reported that treatment with trehalose (likewise with other non-hydrolysable sugars as sucrose) induces vesicle formation in several cell lines, mainly but not exclusively autophagosomes. $^{22}$ 
A
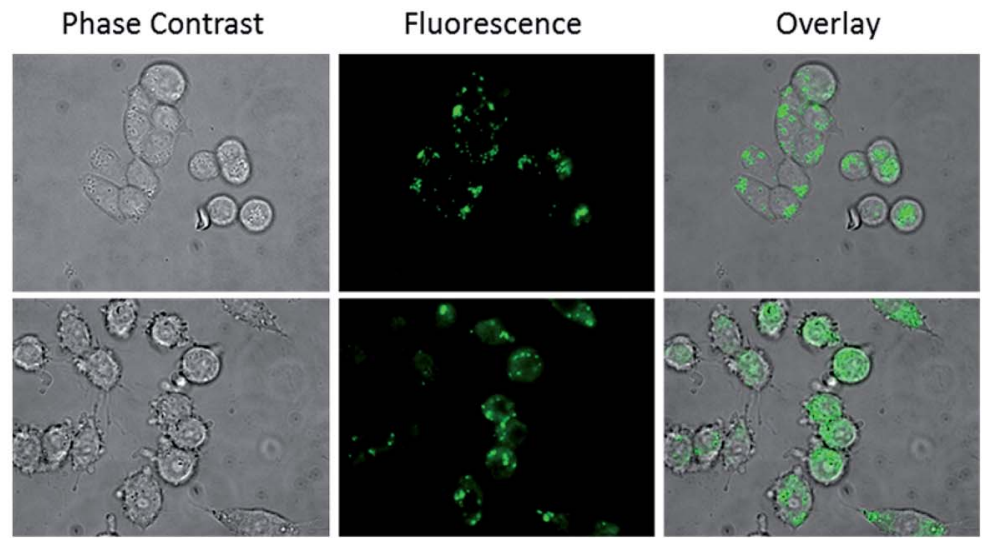

LoVo

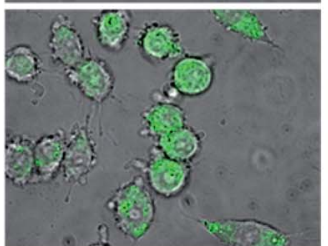

RKO

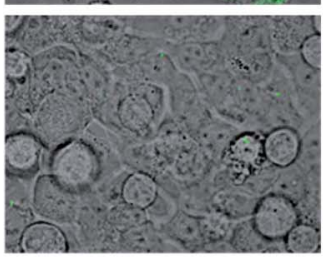

HCT116
B
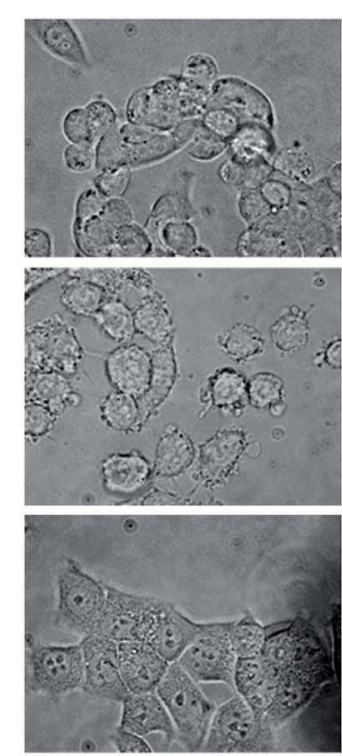

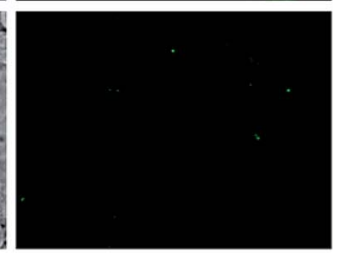

Fluorescence
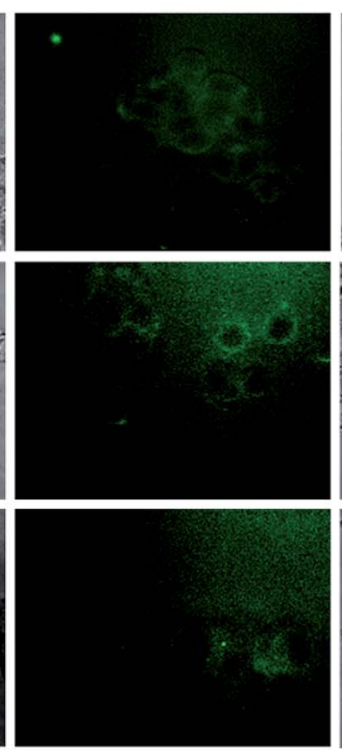

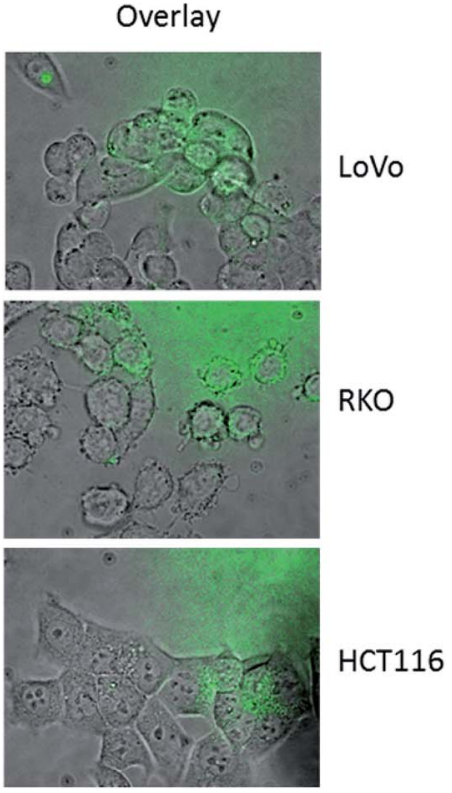

Fig. 3 DAT and DNS-Cl intracellular localization. Representative images of LoVo, RKO and HCT116 cells incubated with DAT (A) or DNS-Cl (B) 1 $\mu \mathrm{M}$ for $30 \mathrm{~min}$ and evaluated in phase contrast and epifluorescence (60× magnification, exposure time $500 \mathrm{~ms})$ microscopy. Overlay images represent the merge between phase contrast and fluorescence pictures.

Hence, to trigger vesicle formation, we treated colon cancer cells with $100 \mathrm{mM}$ trehalose for 16 hours before DAT addition and then analysed the pattern of its accumulation by fluorescence microscopy. Under these conditions, the accumulation of DAT into intracellular vesicles became more evident in all cell lines, including HCT116 (Fig. 4A). Moreover, when cells were treated with $100 \mathrm{mM}$ trehalose, DAT fluorescence was retained in these vesicles also following incubation with DAT for 16 hours rather than 30 minutes in all the analysed cell lines (Fig. 4B). In order to identify these intracellular vesicles, we incubated cells with $100 \mathrm{mM}$ trehalose for 16 hours and then we stained cells for 30 minutes with DAT and with either LysoTracker Red (LTR), a fluorescent dye specific for lysosomes, or LipidTOX Red (LT), a probe that selectively binds neutral lipids specifically staining lipid droplets. No colocalization was observed between DAT and LTR in all the analysed cell models, indicating that DAT is not accumulated in lysosomes (Fig. 5A).

Conversely, the fluorescent stain of DAT perfectly merged with LT (Fig. 5B), indicating that DAT is preferentially accumulated in lipid droplets in intestinal cells. Interestingly, HCT116 showed less lipid droplets compared to LoVo and RKO cells and they were all DAT-positive, suggesting that the reduced DAT staining found 
A
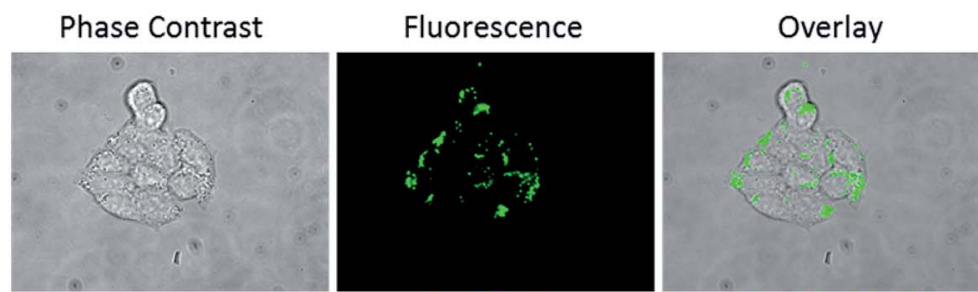

LoVo
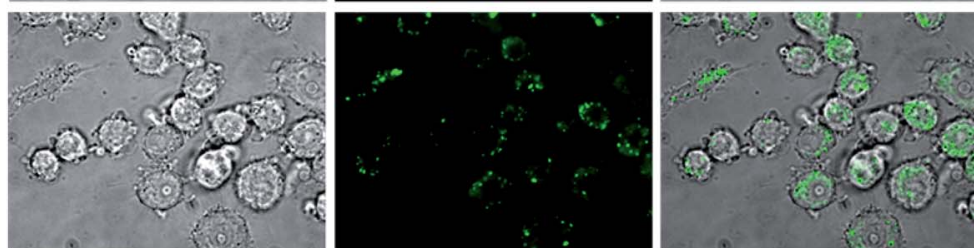

RKO
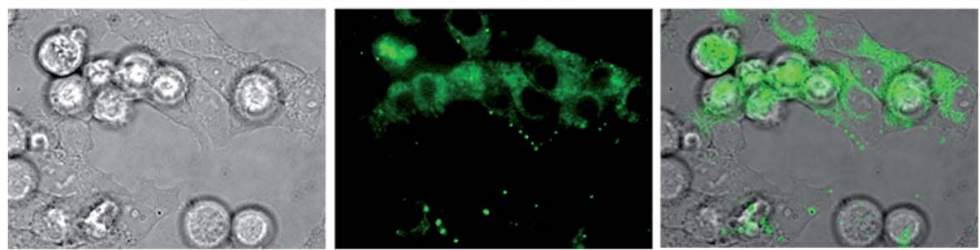

HCT116

B

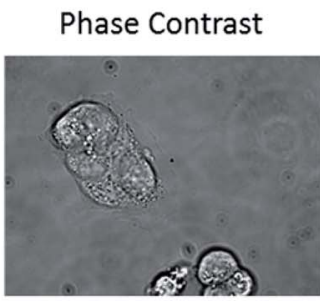

Fluorescence
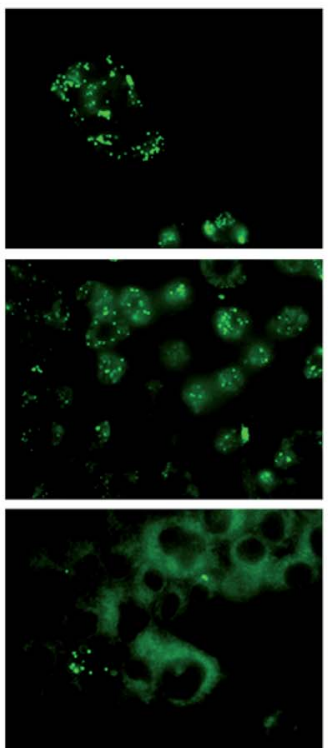

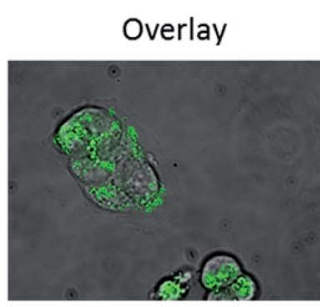

LoVo
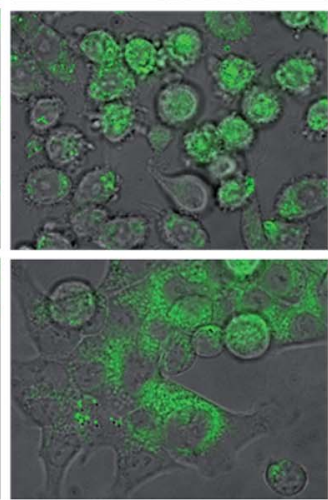

RKO

HCT116

Fig. 4 DAT intracellular localization upon trehalose treatment. Representative images of LoVo, RKO and HCT116 cells upon pre-incubation of $16 \mathrm{~h}$ with $100 \mathrm{mM}$ trehalose and subsequent incubation with DAT $1 \mu \mathrm{M}$ for $30 \mathrm{~min}$ (A) or $16 \mathrm{~h}$ (B), evaluated in phase contrast and epifluorescence $(60 \times$ magnification, exposure time $500 \mathrm{~ms})$ microscopy. Overlay images represent the merge between phase contrast and fluorescence pictures.

in HCT116 cells was not determined by a different uptake of DAT but rather due to a natural propensity of these cells to accumulate less lipid droplets compared to the other colon cancer models. Moreover, compared to LT, DAT produced a clear staining of lipid droplets with a very low intracellular diffuse fluorescence. Overall, these data indicate that DAT is a highly selective dye for lipid droplets and that trehalose internalized within the cell is stored in the lipid droplets compartment.
In conclusion, we report a reproducible synthetic strategy to prepare a fluorescent acetylated trehalose derivative, which exhibits a good permeability in human living cells, maintaining a well detectable intracellular fluorescent signal. Furthermore, this synthesis is cost effective when compared to derivatization with other fluorochromes. The presence of acetyls facilitates the cellular uptake, as shown by the staining pattern obtained in few minutes; as proposed by Abazari, ${ }^{10}$ the cellular esterases are 

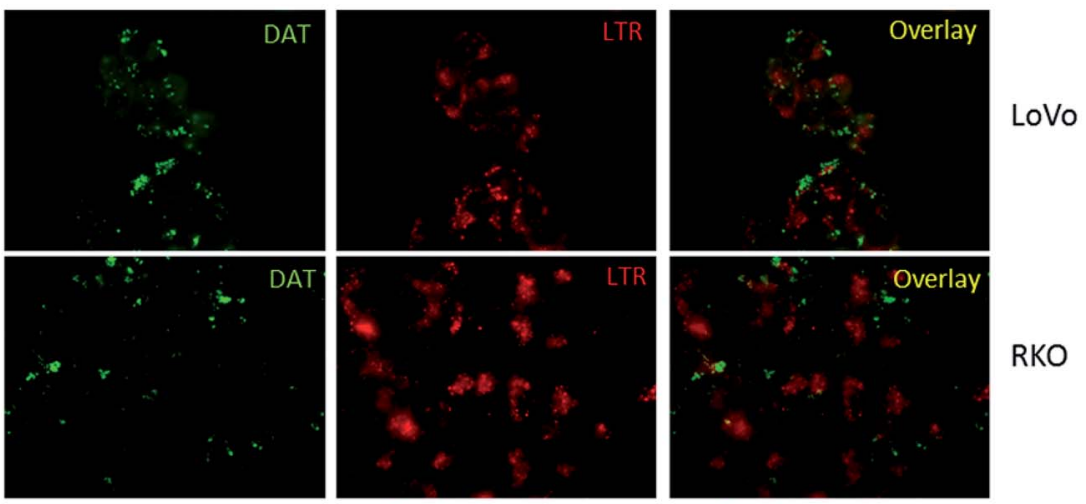

RKO
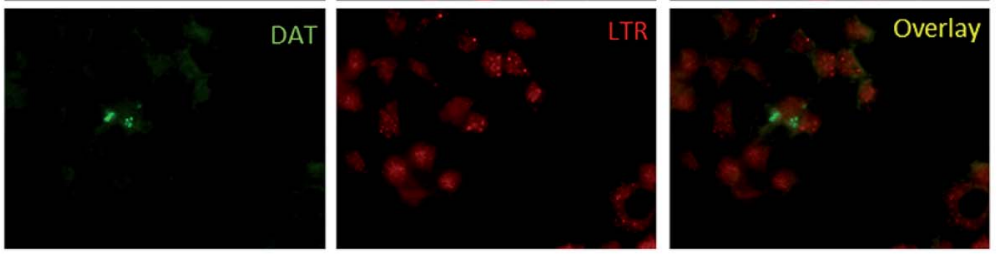

HCT116

B
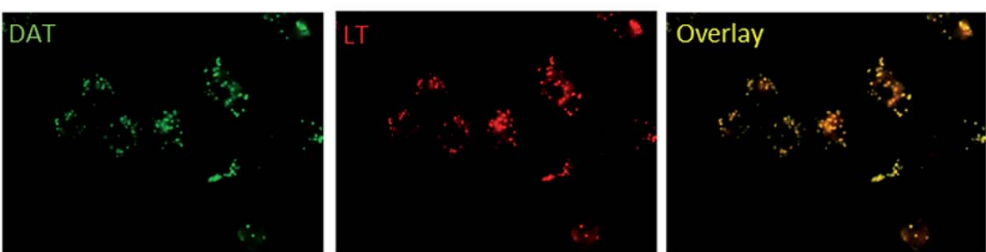

LoVo
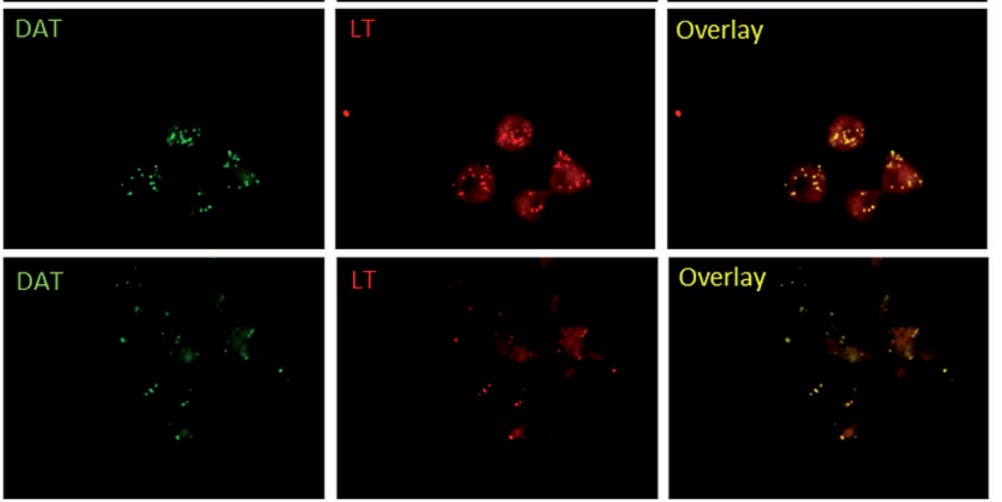

RKO

HCT116

Fig. 5 Identification of DAT-positive vesicles. Representative images of LoVo, RKO and HCT116 cells upon pre-incubation of $16 \mathrm{~h}$ with 100 mM trehalose and incubation for 30 min with DAT and LysoTracker Red (LTR) (A) or LipidTOX Red (LT) (B). The treatments are detailed under Materials and methods in the ESI. $\dagger$ Epifluorescent images were acquired at $60 \times$ magnification with the following exposure times: 500 ms for DAT, 500 ms for LTR and $200 \mathrm{~ms}$ for LT. Overlay images represent the merge of the two fluorescence pictures and show in panel B colocalization between DAT and LT.

expected to rapidly hydrolyze the acetyl groups, thus entrapping the dansyl-trehalose within the cells. Due to the low solubility in water, DAT can be added to cell cultures only at concentrations lower than $5 \mu \mathrm{M}$. However its entrapment within the cells is consistent with a prompt hydrolyzation of the acetyl ester bonds by intracellular esterases, as pointed out by Abazari et al. ${ }^{\mathbf{1 0}}$ The ability of trehalose to rapidly induce the formation of intracellular vesicles is paralleled by the interaction of DAT with these structures, suggesting a common route for native and fluorescent sugar. Finally, DAT fluorescence offers the advantage of being only slightly influenced by the cellular environment, maintaining a good intensity in hydrophobic or hydrophilic compartments.

This is evident from the staining of the tested intestinal cells, were different patterns are recognizable. Notably, DAT is always sequestered in vesicles induced by the sugar treatment, 
indicating that this compound is a promising probe for lipid droplets in living cells. Additionally, when considering that trehalose is an important player in the assembly and architecture of mycobacterial cell envelope, DAT could be profitably used as a fluorescent probe to investigate mycobacterial trehalose processing and trehalose-related mechanisms targeted by novel tuberculosis therapeutics. ${ }^{23}$

\section{Conflicts of interest}

There are no conflicts to declare.

\section{References}

1 L. M. Crowe, Comp. Biochem. Physiol., 2002, 131, 505-513.

2 M. Zhang, H. Oldenhof, B. Sydykov, J. Bigalk, H. Sieme and W. F. Wolkers, Sci. Rep., 2017, 7, 6198.

3 M. Zhang, H. Oldenhof, H. Sieme and W. F. Wolkers, Biochim. Biophys. Acta, 2016, 1858, 1400-1409.

4 K. Deb, Sci. World J., 2009, 9, 236-249.

5 L. Keskintepe and A. Eroglu, Methods Mol. Biol., 2015, 1257, 489-497.

6 P. Loi, D. Iuso, M. Czernik, F. Zacchini and G. Ptak, Trends Biotechnol., 2013, 31, 688-695.

7 A. L. Mayer, C. B. Higgins, M. R. Heitmeier, T. E. Kraft, X. Qian, J. R. Crowley, K. L. Hyrc, W. L. Beatty, K. E. Yarasheski, P. W. Hruz and B. J. DeBosch, Sci. Rep., 2016, 6, 38586.

8 R. Shirakashi, C. M. Köstner, K. J. Müller, M. Kürschner, U. Zimmermann and V. L. Sukhorukov, J. Membr. Biol., 2002, 189, 45-54.

9 T. Uchida, M. Furukawa, T. Kikawada, K. Yamazaki and K. Gohara, Cryobiology, 2017, 77, 50-57.
10 A. Abazari, L. G. Meimetis, G. Budin, S. S. Bale, R. Weissleder and M. Toner, PloS One, 2015, 10, e0130323.

11 K. M. Backus, H. I. Boshoff, C. S. Barry, O. Boutureira, M. K. Ptel, F. D'Hooge, S. S. Lee, L. E. Via, K. Tahlan, C. E. Barry and B. G. Davis, Nat. Chem. Biol., 2011, 7, 228235.

12 M. Kamariza, P. Shieh and C. R. Bertozzi, Methods Enzymol., 2018, 598, 355-369.

13 G. Weber, Biochem. J., 1952, 51, 155-167.

14 R. F. Chen, Arch. Biochem. Biophys., 1967, 120, 609-620.

15 L. Zhang, Y. Li, H. Zhou, L. Li, Y. Wang and Y. Zhang, J. Pharm. Biomed. Anal., 2012, 66, 356-358.

16 R. Nirogi, P. Komarneni, V. Kandikere, R. Boggavarapu, G. Bhyrapuneni, V. Benade and S. Gorentla, J. Chromatogr. B: Anal. Technol. Biomed. Life Sci., 2013, 913-914, 41-47.

17 R. P. Haugland, Handbook of fluorescent probes and research products, Molecular Probes, 2002.

18 D. G. Beach, H. Liu and M. A. Quilliam, Anal. Methods, 2015, 7, 1000-1007.

19 J. Im, S. Kim, Y.-H. Jeong, W. Kim, D. Lee, W. S. Lee, Y.-T. Chang, K.-T. Kim and S.-K. Chung, MedChemComm, 2013, 4, 310-316.

20 X. Ding, W. Wang and F. Kong, Carbohydr. Res., 1997, 303, 445-448.

21 J. R. Lakowicz, Principles of Fluorescence Spectroscopy, Springer US, 3rd edn, 2006.

22 T. Higuchi, J. Nishikawa and H. Inoue, J. Cell. Biochem., 2015, 116, 609-617.

23 A. Nobre, S. Alarico, A. Maranha, V. Mendes and N. Empadinha, Microbiology, 2014, 160, 1547-1570. 